\title{
4H11-28z/fIL-12/EGFRt-expressing Autologous T-lymphocytes
}

National Cancer Institute

\section{Source}

National Cancer Institute. 4H11-28z/fIL-12/EGFRt-expressing Autologous Tlymphocytes. NCl Thesaurus. Code C123823.

A preparation of genetically modified autologous T-lymphocytes transduced with a retroviral vector expressing a chimeric antigen receptor (CAR) targeting the human tumor-associated antigen (TAA) MUC16ecto and encoding the human pro-inflammatory cytokine interleukin-12 (IL-12), fused to the signaling domain of the zeta chain of the TCR/CD3 complex (28z), and a truncated form of the human epidermal growth factor receptor (EGFRt), with potential immunostimulating and antineoplastic activities. Upon intravenous administration, 4H11-28z/fIL-12/EGFRt-expressing autologous Tlymphocytes are directed to and induce selective toxicity in MUC16-expressing tumor cells. In addition, the T-cells secrete IL-12 which induces secretion of interferon-gamma, promotes the activation of natural killer cells (NKs), and induces cytotoxic T-cell responses against tumor cells, which may result in immune-mediated tumor cell death and inhibition of tumor cell proliferation. Devoid of both lig and binding domains and tyrosine kinase activity, the expressed EGFRt both facilitates in vivo detection of the administered, transduced T-cells and can promote elimination of those cells through a cetuximab-induced antibody-dependent cellular cytotoxicity (ADCC) response. MUC16, a transmembrane protein and glycosylated mucin, is overexpressed on the cell surface of the majority of ovarian cancer cells but not on healthy cells. MUC16ecto is the extracellular portion of MUC-16 and is the part that is retained by cells after cleavage of CA-125. 